\title{
Correction to: Transfer Learning of Deep Spatiotemporal Networks to Model Arbitrarily Long Videos of Seizures
}

\author{
Fernando Pérez-García (D), Catherine Scott, Rachel Sparks (D), \\ Beate Diehl (D), and Sébastien Ourselin (1D
}

\section{Correction to:}

Chapter "Transfer Learning of Deep Spatiotemporal Networks to Model Arbitrarily Long Videos of Seizures" in: M. de Bruijne et al. (Eds.): Medical Image Computing and Computer Assisted Intervention - MICCAI 2021, LNCS 12905, https://doi.org/10.1007/978-3-030-87240-3_32

In the first line of Sect. 2.2, "A neurophysiologist (A.A.)" has been replaced by "A neurophysiologist (C.S.)". In addition, Equation (1) and one mathematical expression two paragraphs above this had been rasterized into images. This has been remedied. 